\title{
Justus Von Liebig ¿El creador de un programa de enseñanza de la química?
}

\author{
Justus Von Liebig, is the creator of a program for teaching chemistry?
}

Patricia Pacheco Lozano ${ }^{1}$ y Royman Pérez Miranda²

Grupo de Investigación: Representaciones y Conceptos Científicos. ${ }^{1}$ Estudiante Maestría en Docencia de la Química. Universidad Pedagógica Nacional. Bogotá. ${ }^{2}$ Profesor Departamento de Química. Universidad Pedagógica Nacional. Bogotá. patipalo@yahoo.es, royman@pedagogica.edu.co

\section{Resumen}

La enseñanza de la química se ha venido modificando hace algunos siglos, aunque hay que tener en cuenta que un fuerte cambio en tomo al modo de enseñar química surgió en el siglo XIX, con Justus von Liebig y su programa para la enseñanza de la química a profesionales de esta área en general; dicho programa fue una fusión entre la teoría, la práctica (experimentación) y la investigación en el laboratorio. El resultado de este tuvo una gran acogida por parte de la comunidad química, tanto que hoy en día la gran mayoría de los currículos de formación de profesionales de la química se basan en el método de Liebig.

\section{Palabras clave}

Química, Justus von Liebig, formación, experimentación, laboratorio, enseñanza, didáctica, histórico-epistemológico.

\section{Abstract}

The teaching of chemistry has been changing for some centuries, but it must be borne in mind that a sharp change in the way of teaching chemisty emerged in the XIX century, with Justus von Liebig and his program for the teaching of chemisty to professionals of this area in general, that program was a merger between the theory and practice (testing) and research in the laboratory. The chemistry community very well received the result of this, so that today the vast majority of the curricula of professional training in chemistry are based on the method of Liebig.

\section{Keywords}

Chemistry, Justus von Liebig, training, testing, laboratory, teaching, education, didactics, historical-epistemological.

\section{Introducción}

La enseñanza y el aprendizaje hacen parte fundamental de nuestra vida desde el comienzo, continuamente practicamos el proceso de enseñanza y de aprendizaje; pero, ¿Somos concientes que estos dos procesos se han modificado constantemente? ¿Tenemos presente la forma en la cual el conocimiento científico llegó ha ser transformado e importante para el desarrollo de la química como ciencia y del aprendizaje de la misma?, induso ¿Conocemos quién o quiénes fueron participes de las grandes transformaciones de la química a nivel científico y a nivel de enseñanza? Y ¿Es importante conocer la historia de la formación de profesionales y profesores de la química? Sin duda, estos cuestionamientos han salido a flote a lo largo de la historia de la química como ciencia 
y sus respuestas han sido indagadas, manifestadas y hasta cuestionadas por los diferentes entes de la sociedad (induyendo la comunidad científica especializada), pero, sigue habiendo grandes interrogantes en tomo al acto de aprendizaje y de la misma enseñanza. Al procurar responder estos interrogantes surgen personajes y situaciones nuevas, que llevaron a profundizar en la historia de la química como ciencia de la naturaleza, en la epistemología de la química e induso en la didáctica de la química, además, se produce una búsqueda concienzuda en el interior de siglo XVIII, XIX y $\mathrm{XX}$, en las situaciones que allí se presentaron y permitieron centrarse en las investigaciones y en la forma de enseñanza de Freiherr Justus von Liebig un reconocido químico alemán del siglo XIX, quien fue parte fundamental para el avance y desamollo de la química y de la educación en esta ciencia.

Teniendo en cuenta que esta es una investigación documental de carácter históricoepistemológico de la didáctica de las ciencias, vale la pena informar las características, los hechos y los métodos más relevantes para entender la estructura de los aurríaulos de formación de químicos actuales y áreas afines con la química, las transformación de estos curnículos y, del mismo modo, hacer un acercamiento al programa desarrollado y aplicado por Liebig en su faceta como formador de químicos y entender la importancia y la relación de la experimentación en química con la teoría. Partiendo de la historia social de las ciencias y de la química como un punto de partida esencial, que permite dar la importancia y la relevancia de los hechos que tuvieron importancia hace años y que han trascendido hasta la época actual, contribuyendo a esclarecer inquietudes y métodos de enseñanza.

\section{Aspecto didáctico de las ciencias}

A partir de su conformación como disciplina cient́fica (hacia 1970), la didáctica de las ciencias se ha alejado crecientemente de la tradicional didáctica metodológica presente en la formación del profesorado en ciencias, que estaba estrechamente vinaulada a la pedagogía en muchos países de Europa y Latinoamérica.

La didáctica de las ciencias actual surge más de una confluencia de la actividad en Europa continental con la investigación anglosajona en science education, de naturaleza inicialmente curricular y psicologista, que como una heredera directa de las llamadas didácticas especiales de las distintas ciencias, además, aseguran que la visión de la didáctica de las ciencias es la de una disciplina por el momento autónoma, centrada en los contenidos de las ciencias desde el punto de vista de su enseñanza y aprendizaje (una disciplina de basamento mayormente epistemológico), y nutrida por los hallazgos de otras disciplinas ocupadas de la cognición y el aprendizaje (la psicología y las del área de la ciencia cognitiva) (Aduríz e Izquierdo, 2002).

\section{Aspecto epistemológico de las ciencias}

La epistemología, que sustituyo en el siglo XX a la anterior filosofía de las ciencias, aryo objeto de reflexión lo constituye el análisis del desarrollo del conocimiento científico, de las ideas que se sostienen sobre su origen, fundamentos objetivos, aceptación e importancia. De manera espećfica la constitución de las teorías, para demás programas de investigación o modelos científicos con el intento de explicar y describir la lógica intema y las condiciones extemas que han incidido significativamente en esta constitución (Gallego y Pérez, 2005). Otro criterio de la filosofía (actualmente llamada epistemología) es la idea de que una teoría científica es tanto más perfecta cuanto mayor es su grado de formalización, pero que esa característica sólo tiene sentido cuando se refiere al momento en que un modelo cient́fico goza de plena vigencia y madurez, y no a etapas en que su verificación aun es incipiente o cuando ya comienza a entrar en decadencia. También la epistemología ha elaborado principalmente un discurso teórico sobre la ciencia considerándola 
como una forma de conocimiento cuyo objetivo consiste en indagar la estructura lógica de las leyes que rigen los fenómenos de la naturaleza. A partir del conocimiento de esas leyes, la ciencia construiría modelos explicativos más amplios como son las teorías cient́ficas con una reconocida capacidad de explicar la realidad y predecir el desarrollo de cuanto en ella acontece (Barona, 1997).

\section{Aspecto histórico de las ciencias}

La Historia de la ciencia es el campo de la historia que estudia el desarrollo temporal de los conocimientos cient́ficos y tecnológicos de las sociedades humanas, lo anterior está relacionado con el impacto que la ciencia y la tecnología han tenido históricamente en la cultura, la economía y la política. Eslava, J. (2004) formula un indicio paradójico diciendo: la historia de la ciencia cobra vida propia y reconocimiento social, en el momento en el cual se considera que para el desarrollo de la ciencia, y para la formación científica del científico, el reconocer la historia de cada disciplina no proporciona elemento alguno de relevancia para el quehacer cotidiano al interior de la ciencia. Es acá donde la historia de la ciencia recurre al método histórico tanto de la historia intelectual como de la historia social, a partir de esto se empieza a hablar de historia social de las ciencias, teniendo en cuenta que esta ve la historia a través del análisis de los sucesos históricos, epistemológicos, didácticos, sociales, económicos y políticos, para poder ver su coherencia lógica, los contextos y además comprender las condiciones dominantes. Asimismo, esta permite dar auenta sobre la historia social de la química, la formación en química y las propuestas de los diferentes actores de estos sucesos. La gran distinción entre la manera de procedimientos en química y la naturaleza filosófica es, que una es un peso y la otra es una medida. La filosofía natural se aplicaba en las medidas para la naturaleza por muchos siglos, pero sólo por cincuenta años debemos procurar avanzar en nuestro pensamiento filosófico, todos los grandes descubrimientos químicos son debidos a la "balanza" -el incomparable instrumento que da permanencia a cada observación, disipa toda ambigüedad, estableciendo la verdad, detectando errores, y nos dinige en la parte verdadera de la ciencia inductiva (Liebig, 1859).

Acudiendo a una versión de la historia de las ciencias de la naturaleza, se ha venido argumentando que la didáctica de las ciencias es, en la actualidad, una disciplina cient́fica, argumentos que son los que demuestran que hay una comunidad de especialistas que ha formulado sus problemas y, por tanto, sus respectivos campos de investigación; que publica los resultados obtenidos en un creciente número de revistas especializadas; $y$, que se congrega en encuentros, simposios, congresos y demás (Pérez, Gallego, y colaborador 2007).

Teniendo en auenta que desde la química como ciencia se puede estudiar la formación en química con una perspectiva histórico epistemológica, cabe destacar la labor de los científicos y de todos aquellos investigadores que contribuyeron a fortalecer las ciencias, y especialmente a la química como una ciencia de la naturaleza y que además permitieron introducir cambios y diferentes modelos que contribuyeron a la creación de un programa estructurado de enseñanza.

De este modo se puede afirmar que la historia de la química generalmente se relaciona con la historia de los químicos, donde ellos han hecho progresos significativos a la química como ciencia experimental, ya sea de forma individual o en un constructo colectivo, esto se reconoce con los principales acontecimientos que marcan el nacimiento de la química. A partir de esta se crea la necesidad de reinventar los laboratorios y la forma en la que se aprendía la química, además se crean las primeras comunidades organizadas "comunidades científicas". Sin dejar de lado que estos científicos se ceñían a la conciencia social, a los partidos políticos y al desarrollo según su época. Cada uno de los científicos ha sido parte importante en la creación y desarrollo de la química, entre algunos científicos destacados encontramos a George Emst Stahl, Claude Louis Berthollet, Henry Le Chatelier, Carl Withelm Sheele, Priestley, Mendeleev, F.A. Kekulé, Justus Von Liebig, entre otros. 


\section{Enseñanza de la química teórico-práctica}

La ciencia es una fuente importante para las comunidades científicas (de químicos), y su participación a través de la historia, en donde se han formado profesionales preocupados por mejorar su disciplina para enseñar las ciencias (induyendo la química), por medio de la creación de programas, los auales tenían unos procedimientos y estructuras definidas, siendo para esta investigación primordial el programa propuesto por Justus Von Liebig, y los aportes que el hizo al desarrollo de la química como ciencia.

Liebig no fue el primero en desarrollar un laboratorio en el cual los estudiantes experimentaban (Good, 1936). La enseñanza de laboratorio estaba allí antes de Liebig, pero su enfoque pedagógico en química era nuevo (Sachtleben, 1958). Antes que el estableciera el laboratorio allí, no había un verdadero laboratorio de enseñanza de química en Alemania y muy pocos en aualquier parte, y es quizás uno de sus más importantes servicios a la química que promovió la idea que la experiencia de la práctica sistemática es vital para el estudio de una ciencia (Twigg, y Twigg, 1973). Y aunque Liebig no invento la enseñanza en el laboratorio, él estaba presente en el momento histórico en que permitió y mereció la explotación. No sólo estaba él allí en el momento oportuno sino que también era un promotor muy competente, de hecho, él tuvo que superar las dificultades en medio de las cuales se encontraba (Good, 1936). Durante el siguiente cuarto de siglo liebig se agotó por hacer de Giessen la principal institución para la instrucción química en el mundo. No obstante de un escaso salario e inadecuadas asignaciones, él llevo la instrucción práctica en química a cierto nivel que los estudiantes atraídos a la universidad no sólo eran de Alemania, sino de Francia, Inglaterra, Italia, Estados Unidos y México (Inde, 1964). El comienzo de la instrucción de laboratorio en química o en otras ciencias, es difícil de dar un dato con exactitud, como la invención de la imprenta; y por la misma razón, esto comenzó con un proceso gradual al cual muchos trabajadores independientes sin nombre, hicieron contribuciones antes de que alguien tuviera conocimiento de su desarrollo (Good, 1936).

Según Brock, W. (1998) hacia 1820 fue la época en que Thomas Thomson estaba creando un laboratorio para la enseñanza práctica de la química en Glasgow. Otros han considerado a Thomas Thomson como el pionero en abrir el laboratorio químico a estudiantes. En 1817 fue el año de Thomson para el nombramiento de la recién creada cátedra de química de la Universidad de Glasgow y un año más tarde él fue nombrado Profesor Regius. Pero Thomson había enseñado química en Edimburgo en un periodo anterior. Walter Crum, al dar cuenta de este tiempo, escribió: "Dr. Thomson (comenzando in 1801) continuó dando conferencias en Edimburgo hasta 1811, y durante este tiempo él abrió un laboratorio para pupilos, probablemente el primero establecido en Gran Bretaña" (Good, 1936). Cuando Justus von Liebig fue nombrado a los veintiún años profesor ayudante de química en la pequeña y aletargada Universidad de Giessen, a unos treinta y siete kilómetros al norte de Frankfurt, aspiraba conseguir fama y fortuna estableciendo una escuela privada de farmacia más que con la enseñanza de la química en la universidad. J. B. Trommsdord (1770 - 1837) había hecho lo mismo, con éxito en la Universidad de Erfurt, donde también dirigía una escuela privada de farmacia. Al final, aunque un gran número de alumnos de Liebig fueron destinados a boticas de Alemania Liebig y Giessen se hicieron celebres como ejemplo de una institución dedicada a la enseñanza de la química práctica (Brock, 1998). El siguiente es un fragmento de una carta a Schleiermacher, Giessen, Julio 30, 1825, "Usted podrá ver desde un prospecto encerrado que el profesor Wemekingk. Umpfenbach, y yo estamos unidos para establecer un instituto químico-farmacéutico. En Alemania sólo hay dos institutos como tal; un en Erfurt bajo la dirección del Profesor Trommsdorf; y el otro en Jena, dirigida por el Profesor Göbel". "El número de quienes quieren aplicar cada año como hombres nuevos al instituto es tan grande que ambos pueden aceptar difíalmente un-sexto de ellos. El número de los que son aceptados es seis de veinte. Yo creo que nosotros juntos podemos con 
muchos más, que sí uno solo dirigiera el instituto, porque un personal interesante puede ser incrementado considerablemente por una acción conjunta. Nosotros no tenemos duda de todos los grandes sucesos, especialmente desde que el profesor Schmidt ha resuelto las conferencias de física. Para Giessen y la universidad esto no es sin fama. Esto siempre hará que se incremente el número de estudiantes alrededor de veinte o treinta" (Berl 1938). Como hemos visto, esto se consiguió principalmente gracias a que liebig dirigía un instituto farmacéutico privado, independientemente de su actividad docente estatal en la universidad, aunque debió de haber alguna coincidencia en sus actividades. Desafortunadamente, no nos han llegado detalles acerca del número de alumnos, o de dónde se desarrolló esta actividad privada, ni del éxito financiero que pudo tener. Lo único que se sabe es que el instituto consiguió atraer a alumnos de todos los estados alemanes y, lo que es más significativo, del extranjero (Brock, 1998).

Cuando Liebig comenzó su trabajo en Giessen no tenia laboratorio disponible para él, y sabia por su experiencia personal que un aurso de química con trabajo práctico era una esperanza vaća (Oesper, 1927). Liebig requirió que sus estudiantes condujeran experimentos reales en el laboratorio desde el primer día de sus estudios, para induir en ensayos prácticos de todos los variados análisis cualítativos y cuantitativos, y actualmente para preparar compuestos orgánicos e inorgánicos. Esta clase de estudio es mantenido esencialmente a este día. Pero se ha extendido e intensificado para abrazar otras ramas de la química (Scharrer, 1949).

El laboratorio de Giessen, ubicado en unos barracones abandonados y sin calefacción fue el más famoso del mundo en la enseñanza práctica del análisis químico y muy en especial por el uso de un método de análisis orgánico de éxito seguro. Tanto éxito obtuvo que en 1839 ya se podía hablar no de laboratorio sino de laboratorios (Brock, 1998). Aunque la declaración de Lord Kelvin que "todos los químicos eminentes, que eran jóvenes en 1845 eran estudiantes de Liebig" es una gran exageración, no obstante es verdad que el laboratorio de Giessen era el centro más influyente en donde el nuevo método de enseñar química se extendía (Good, 1936).

Ahora e posible explicar el modo en que Liebig creó una gran escuela en enseñanza e investigación, que se convirtió en modelo para otras en Alemania y en el extranjero. En un artículo fundamental, J.B. Morrell apuntó que las condiciones que confluyeron en el éxito de Liebig eran de índole intelectual, institucional, técnica, psicológica y financiera. En primer lugar, Liebig tenía un programa de investigación (el análisis de los compuestos orgánicos) y otro docente (la enseñanza práctica del análisis cualitativo y cuantitativo) precisos. En 1831 Liebig había alcanzado una reputación nacional e internacional más que suficiente para el desarrollo de estos programas afines, mientras que sus Annalen iban a convertirse en órgano de expresión suyo y de sus alumnos. Esta reputación, junto con la convicción creciente de que tenía unos conocimientos y unas técnicas que impartir, ayyo estudio podía ser suficientemente útil para el desarrollo de la farmacia y de la medicina, hicieron que en la década de 1840 la enseñanza de la agricultura y de la química garantizaran el cumplimiento de otra de las condiciones necesaria para el éxito: la existencia de un número suficiente de alumnos (Brock, 1998). Él y sus estudiantes hicieron muchas contribuciones experimentales, particularmente en el área de química analítica, orgánica y agricultura. Su gran energía y entusiasmo lo hicieron un profesor inspirador y un prolífico escritor. Al mismo tiempo él fue un vigoroso propagandista y un impulsivo dogmatista. Por un tiempo él logró la posición autoritaria antiguamente retenida por el ahora envejecido Berzelius (Inde, 1964). El rápido y preciso método de análisis de Liebig, no sólo supuso el secreto de su triunfo personal como profesor, sino que condujo a una explosión de actividad y a una inmensa clarificación de la evidencia química: por primera vez se veían las relaciones entre compuestos orgánicos y se podía hacer una dasificación de las especies. El bosque inextricable de Wöhler se había transformado en un jardín botánico (Brock, 1998). Desde 1824 a 1852, Liebig dirigió un laboratorio químico en la Universidad de Giessen en Alemania, en el aual cientos de estudiantes se reunieron (Sheppard, 2006). Una carta para August Walloth, Giessen, septiembre 23 De 1824, dice: "Imagínese, comprando instrumentos, reactivos, $y$ materiales para laboratorio, pude obtener una donación de 
cien florines después de grandes dificultades. iPude tener la misericordia divina para míl ¿Cómo pude dirigir con tan poco? Pero yo pude resistir a todo esto si sólo podía economizar al final con el falso razonamiento de Zimmermann (1782-1825) y su claque. Ellos amargaron mi vida aquí $y$ estropearon a su antojo. Yo use todo mi tiempo para la preparación de mis conferencias, Ilenando mis documentos, atendiendo a fiestas y bailes, y visitando al Jardín de Arbustos. Esto, en unas pocas palabras es lo que puedo agregar: permitiéndome tener buenas noticias de usted pronto, $y$ no castigándome aunque lo merezca. Usted sabe que un hilo de paciencia es una cadena de hierro, pero la mía es tomada como una telaraña"(Berl 1938).

Liebig se encontraba en una continua y amargada lucha con la petulancia de la universidad, la cual exigió dejar certificado y, de hecho el clásico, uno como el prerrequisito indispensable para la admisión a la universidad y a estos institutos, el cual, para estar seguros, el laboratorio al que también pertenecía (Good, 1936). Sin embargo, el talento de Liebig y su gran personalidad hacia que impresionara a sus compañeros docentes que después de la muerte del profesor de química ordinario, Liebig fue nominado para cubrir la vacante y el único voto contrario fue el del profesor de hebreo (Oesper, 1927).

En 1833 y como resultado de sus negociaciones con el gobiemo, Liebig unió su escuela privada y su curso oficial de la universidad; para entonces sabemos que tenía entre diez y quince estudiantes de farmacia, y entre tres y cinco estudiantes de química al año. Durante las dos últimas décadas siguientes el número de alumnos que estudiaban química superó al de los estudiantes de farmacia (Brock 1998):

\begin{tabular}{|c|c|c|c|c|c|}
\hline & $1830-35$ & $1836-40$ & $1841-1845$ & $1846-50$ & TOTALES \\
\hline Química & 15 & 75 & 174 & 143 & 407 \\
\hline Farmacia & 53 & 63 & 74 & 62 & 252 \\
\hline
\end{tabular}

Los estudiantes de muchos países vinieron a Liebig durante el periodo de 1825-1852, comenzó con dos estudiantes, pero el laboratorio pronto fue desbordado y tuvo que ser ampliado. Con el tiempo, los principiantes tuvieron que ser supervisados por sus asistentes y tuvieron que progresar por medio de una serie de ejercicios de laboratorio. El uso de la balanza y los aparatos usuales tuvieron que ser aprendidos en el orden en que las incógnitas podían ser analizadas. "EI conocimiento de la composición de los compuestos permite al químico solucionar problemas químicos, lo cual se mantuvo insoluble sólo unos pocos años," declaro Liebig (Sachtleben, 1958). Las conferencias fueron, sin duda, no modelos, sino donde se consideraban las descripciones, la eficiencia de los experimentos o la derivación de las conclusiones y de las inferencias (Good, 1936). Los éxitos de Liebig como profesor fueron inmediatos. Su amplio conocimiento, su habilidad en el laboratorio. Su entusiasmo, su habilidad para superar dificultades, la facilidad con que analizaba problemas, ganándose el respeto y admiración de sus estudiantes. Su capacidad para trabajar, en la práctica y en la literatura, parecía ilimitada y demandaba y recibía la sincera cooperación de los que trabajaban con él (Oesper, 1927). El éxito de Liebig en atraer estudiantes y desarrollar una reputación universal resultó de sus logros investigativos, de su desarrollo de un concreto y fructífero método de instrucción y, la difusión de su metodología por los leales y devotos estudiantes (Sheppard, 2006). Sus estudiantes, incluso, en el redescubrimiento que era ya familiar, trabajaban con la sensación que estaban encontrando algunas cosas nuevas; y en ese momento donde ellos estaban dirigidos a cuestiones de hecho y a las deducciones que se pueden extraer de ellas, ellos sentían el estímulo a seguir y a hacer investigaciones independientes (Good, 1936). En adición a estos logros investigativos, Liebig fue también reconocido como un educador, en cuyo laboratorio los químicos recibían una educación práctica rigurosa (Sheppard, 2006). Pero el poseía el más alto grado de poder de despertar las más altas habilidades investigativas de sus estudiantes, para estimularlos en la solución de problemas y enigmas y en caminos aparentemente indirectos, para guiarlos a los caminos que los llevarían a sus objetivos (Good, 1936). 
En Giessen formó un grupo distinto en la comunidad; no tenía el tiempo o el deseo para las frivolidades del estudiante ordinario. Cada uno fue inspirado para hacer lo mejor "si quieres ser un químico, tendrás que arruinar tu salud; nadie que no amuine su salud con estudio nunca hará nada en química actualmente" fue el consejo de Liebig para Kekulé. El estudiante, después preparó los más importantes gases, fue cuidadosamente entrenado en análisis cuantitativo y cualítativo... Liebig estableció gran tensión es esta parte de la instrucción, porque él creía que sólo de este modo podía cada uno familiarizarse con las propiedades de las sustancias; en pocas palabras, esta era la única manera de convertirse en químico (Oesper, 1927). En las conferencias experimentales, constantemente se ocupaba de los materiales incorrectos o acertados, sólo para que los asistentes en la mano derecha y la izquierda colocarán los instrumentos y reactivos adecuados (Good, 1936).

Al final de la primera semana Liebig anunció que cada sábado en la tarde, se llevaría a cabo un examen semanal, al cual invitaría a todos aquellos que tuvieran un interés serio en la química. En el primer examen, el salón de conferencias estaba lleno. Él evaluó muy rigurosamente, sondeando los fundamentos mismos de los temas, y a cualquiera que no pudo responder lo dejó sentado con su boca abierta. Para la segunda prueba había, sumando a quienes trabajaban en el laboratorio, sólo alrededor de una veintena de personas; y para la tercera los estudiantes de laboratorio tampoco aparecieron - inosotros éramos cinco hombres, en total Liebig con una sonrisa maliciosa dijo: i"Ahora la paja se ha separado del trigo! Nosotros no vamos a seguir con el examen, caballeros. Sin embargo, si yo veo que ustedes tienen un serio interés en la ciencia, yo los invito a entrar a mi laboratorio. Usted puede tener un lugar allí. Ustedes deberán elegir en relación con el calendario de sus otras dases los días y las horas en las que deseen trabajar en el laboratorio. Mi asistente el señor Ettling, les dará las instrucciones necesarias y pueden ser de utilidad para ustedes en sus primeros esfuerzos". Ettling, el asistente, era un profesor modelo de todas las habilidades manuales. (Good, 1936). Evidenciados del adiestramiento indicado que los estudiantes empezaban por aprender las técnicas fundamentales, afilando un auchillo, cortando y perforando corcho, soplando y curvando vidrio, bajo el ojo vigilante de Carl Ettling (1806-1856), uno de los asistentes de Liebig (Sheppard, 2006). Después de este primer entrenamiento, al estudiante le era permitido participar en la investigación original. El énfasis estuvo siempre en la enseñanza de los estudiantes a observar y razonar independientemente. Liebig créa que la química no era sólo una ciencia y una profesión; esta era también un arte que debía permitir el juego libre de autosuficiencia individual (Oesper, 1927). Los estudiantes de Liebig e induso Liebig mismo permanecían en el laboratorio y sus vidas giraban entorno a este. Quienes estudiaban la química exclusivamente formaban un distinguido clan en la Universidad de Giessen. Trabajaban en el laboratorio, desde temprano en la mañana hasta la noche con la excepción del sábado en la tarde cuando el frecuentemente llamado servidor, Aupel, asistía a hacer la limpieza necesaria de las habitaciones. Pero en todos los otros días de la semana el trabajo se hacía sin interrupción y Liebig por si solo era capaz de mantenerlos ocupados. De este modo se vivía y casi se movía exclusivamente en una sociedad de químicos (Good, 1936).

Liebig es el pionero en el concurso de premios en la química y es probable que el concurso fuese la entrada al salón de conferencias o incluso al laboratorio, Liebig en el anuncio escribió; " "Si bien la química más que cualquier otra ciencia, aseguran estos quienes perseguían los estudios con anhelo, con una merecida recompensa, aunque la distinta utilidad de este conocimiento, yo decidí el confirmar el reconocimiento de la excepcional industria de mis estudiantes presentes en un camino inusual... A todas las preguntas se les dará un número, la magnitud del aval puede indicar la dificultad de la pregunta. Quienes dan la respuesta correcta lo más cercanamente, los cuales serán determinados para la suma de los números de las preguntas resueltas correctamente, pueden ser los merecedores de dos premios". Se sabe que el primer premio era un cuchillo químico (espátula) - con una hoja de platino y paladio y soportes de plata. El segundo premio es una lámpara química (Schierz, 1929). 
Todos los detalles del método de Liebig se publicaron en 1839 en un manual para estudiantes titulada Instructions for the Analysis of Organic Bodies. Este, como la mayoría de los libros de Liebig apareció simultáneamente en alemán y en ingles. El método resulto tan perfecto, que la segunda edición, en 1853, sólo fue necesario introduair muy pocas modificaciones. Para entonces su discípulo y traductor A. w. Hofmann ya había comenzado a experimentar con gases como fuente de calor, con el objeto de sustituir la lámpara de alcohol. Dejando a un lado la adopción del mechero Bunsen en la década de 1860, el método de Liebig permanece aún vigente (Brodk, 1998). En gran parte debido a su escuela, la química orgánica se convirtió en un gigante entre las ramas de la química. "Cuando es posible encontrar un punto brillante en el área turbia de la naturaleza orgánica, que nos parece como uno de los portales a través del cual podemos quizás golpearnos con la verdadera trayectoria para la comprensión y el estudio de esta región, siempre podemos sentir que tenemos razones para felicitamos a nosotros mismos," escribieron Liebig y Wöhler (Sachtleben, 1958). De hecho los químicos que no se habían formado en Giessen sólo encontraron el método de Liebig más sencillo y efectivo después de mucho investigar a fondo (Brodk, 1998).

\section{Bibliografía}

Aduríz, A. e Izquierdo, M. (2002). Acerca de la didáctica de las ciencias como disciplina autónoma. Revista electrónica de enser̃anzas en ciencias. 1 (3).

Barona, F. (1997). Metodologías y técnicas para la investigación social e histórica. Colombia: Corporación autónoma de occidente.

Berl, E. (1938). Justus Liebig, May 14, 1803 - April 18, 1873. Joumal of chemical education. 553 $-562$.

Brock, W. (1998). Historia de la química. Madrid: Alianza editorial.

Eslava, J. (2004). Tensiones y confluencias. Una mirada fugaz al triple legado de los estudios históricos sociales sobre la ciencia. Revista Colombiana de Sociología 23, 159-180.

Gallego, R y Pérez, R. (2005). La formación inicial de profesores de ciencias en Colombia. Colombia. Universidad Pedagógica Nacional.

Good, G. (1936). On the early history of Liebig's laboratory. Joumal of Chemical Education. Diciembre, $557-563$.

Inde, A. (1984), The Development of Modem Chemisty. New York; Courier Dover Publications.

Liebig, Justus. Von. 1859. Familiar letters on chemisty, in its relations to physiology, dietetics, agriaulture, commerce and political economy. Cuarta edición. Londres: Editorial Walton And Maberly, Upper Gower Street, \& Ivy Lane, Paternoster Row.

Oesper, R. (1927). Justus Von Liebig - Student And Teacher. Joumal of Chemical Education. 4 (12), 1461-1476

Pérez, R, Gallego, R. y Claborador. (2007). La formación inicial de docentes de ciencias en nueve universidades suramericanas. TED. Número extraordinario.

Sachtleben, R. (1958). Nobel Prize Winners Descended From Liebig. Journal of Chemical Education. 35 (2), 73-75.

Schierz, Emest R. (1929). Justus von Liebig: The Sponsor of a Prize Contest in Chemistry. Joumal of chemical education. 6 (5), 973-976.

Sheppard, Keith. 2006. From Justus von Liebig to Charles W. Eliot: The Establishment of Laboratory Work in U.S. High Schools and Colleges. Joumal of Chemical Education 83 (4), 556570.

Twigg, C. y Twigg, M. (1973). Centenary of the Death of Justus Von Liebig. Joumal of Chemical Education. 50 (4), 273-274 